\title{
Study of the bullwhip effect in Chinese coal supply chain under fuzzy environment
}

\author{
Xiao Hong Yun ${ }^{1,2, a}$, Jin Suo Zhang ${ }^{3}$ \\ ${ }^{1}$ School of management, Xi'an University of Science \& Technology, Xi'an, 710054, China \\ ${ }^{2}$ Research center for energy economic and management, Xi'an University of Science \& Technology, Xi'an, 710054, China \\ ${ }^{3}$ School of management, Yan'an University, Yan'an, 716000, China
}

\begin{abstract}
Supply chain management is important for coal companies and organizations to improve their business and enhance competitiveness in the Chinese marketplace. The bullwhip effect problem of coal supply chain systems with all demands, lead times, and ordering quantities in an uncertain environment is addressed in this paper. To simulate the bullwhip effect, the Hong Fuzzy Time Series approach and Genetic Algorithm module are preferred as a superior forecasting model. And then a back propagation Neural Network module is added to defuzzify the output of the proposed model. So the bullwhip effect is calculated and analyzed here. The effectiveness and flexibility of proposed method is verified through simulation study.
\end{abstract}

Keywords: Supply chain management, bullwhip effect, coal production, uncertainty.

\section{Introduction}

China is now the largest energy consumer in the world. Providing sufficient coal supply to meet the requirements of a huge population with rising living standards will be a difficult task. The Chinese government is now facing severe challenges from a coal supply problem. With increasingly competitive coal market and high coal consumption, the coal supply chain has become critical and strategic to long-term development in China.

In coal supply chains, multiple independent mines are often connected to a shared rail network. A single rail operator often coordinates rail schedules with the mines, to schedule trains between the mines and the transport terminal in order to meet transport demand of each mine. A common phenomenon in the coal supply chain management that has been observed and justified is known as "bullwhip effect" [1]. Lee, Padmanabhan, and Whang have identified five major causes for bullwhip effect in the supply chain. They are listed as demand forecasting, order batching, price fluctuations, supply shortages and non-zero lead-time ${ }^{[2]}$. According to Sterman, the bullwhip effect originates from the non-optimal solutions adopted by a supply chain participant while does not consider the supply system as a whole ${ }^{[3]}$.

Among various causes of the bullwhip effect, forecasting methods are considered as one of the most important causes because the inventory system of a coal supply chain is directly affected by the forecasting method. Many scholars have tried to quantify and eliminate this effect. Graves quantifies the bullwhip effect for a supply chain in which the demand pattern follows an integrated moving average process ${ }^{[4]}$. Zhang investigates the impact of different forecasting methods on the bullwhip effect for a simple inventory system with a first-order autoregressive demand process ${ }^{[5]}$. Chen et al. show that increasing lead time enhances the bullwhip effect regardless of the forecasting methods employed $^{[6]}$. The authors considered a supply chain as a business process which had to be redesigned assuming that the main cause of the bullwhip effect was the absence of coordination in the management of the supply chain. Following a systems analysis approach, Chen and Disney were able of reducing the bullwhip effect by controlling the order policy by using a proportional controller ${ }^{[7]}$. An improvement in the cost saving due to the reduction of order variance was obtained. Lee et al. measure the benefit of information sharing between a retailer and a manufacturer in a two-stage supply chain in which the demand pattern follows a first-order autoregressive process and an order-up-to policy with a minimum mean square error forecasting technique is used at the retailers ${ }^{[8]}$.

As a matter of fact, for analyzing the bullwhip effect in the real world, few scholars consider it in a fuzzy environment. Some feasible methods have been proposed in supply chain management researches, and most of them focus on controlling the uncertainty causes of bullwhip effect. Pishvaee et al. proposed a robust optimization model and relative robust counterpart for handling the inherent uncertainty of input data in a closed-loop supply chain network design problem ${ }^{[9]}$. The widely used time series models for forecasting purpose especially ARIMA model is generally applicable to linear modeling but it hardly captures the non-linearity inherent in time series data.

In this paper, Fuzzy Theory is utilized as an analysis method to represent and interpret the uncertainty in the coal supply chain management. The authors try to study

\footnotetext{
${ }^{\mathrm{a}}$ Corresponding author: yun_xiaohong@163.com
} 
the effect of fuzziness, i.e., information uncertainty, on the bullwhip effect. Here, it is assumed that all demands, lead times and order quantities have fuzzy values, i.e., they are imprecise. Then, the bullwhip effect is simulated in such an environment. Firstly, to simulate the bullwhip effect in a fuzzy environment, the Hong Fuzzy Time Series approach and Genetic Algorithm module are preferred as a superior forecasting model. Next, a back propagation Neural Network module is added to defuzzify the output of the proposed model. In addition, the bullwhip effect is calculated and analyzed. Finally, we discussed the validation of the proposed solution approach.

\section{Proposed Model and algorithm}

\section{Fuzzy time series model}

Here we explains the time variant fuzzy time series model by Hong ${ }^{[10,11]}$. Let $\xi$ be the class of all fuzzy quantities defined on $[0, \infty]$ which are continuous non-increasing with the strict maximum 1 in the point 0 , where its members are called shapes. Let $\mathrm{N}$ be a LR-fuzzy number whose membership function is defined as follows:

$$
N(x)= \begin{cases}L((m-x) / a) & x \leq m, a>0 \\ R((x-m) / b) & m>x, b>0\end{cases}
$$

where, $L, R \in \xi$. Symbolically, $N=(m, a, b)_{L R}$, respectively.

Let $T_{w}$ denote the weakest t-norm defined by:

$$
T_{w}(x, y)=\left\{\begin{array}{cc}
\min (x, y) & \text { if } \max (x, y)=1 \\
0 & \text { otherwise }
\end{array}\right.
$$

(2)

Let $A_{i}=\left(m_{i}, L_{i}, \quad R_{i}\right)$ be LR-fuzzy numbers, then weakest sum and minus showed by $+w$ and $-w$ are defined as:

$$
\begin{aligned}
& A_{1}+{ }_{w} A_{2}=\left(m_{1}+m_{2}, \quad \max \left\{L_{1}, \quad R_{2}\right\}, \quad \max \left\{R_{1}, L_{2}\right\}\right) \\
& A_{1}-{ }_{w} A_{2}=\left(m_{1}-m_{2}, \quad \max \left\{L_{1}, \quad R_{2}\right\}, \quad \max \left\{R_{1}, L_{2}\right\}\right)
\end{aligned}
$$

(4)

Let $F(t), t \in T$ be a fuzzy time series with the observation $F(t)=(m(t), \quad L(t), \quad R(t)) \quad$ as fuzzy numbers. Then,

$$
D^{k} F(t)=D^{k-1} F(t+1)-_{w} D^{k-1} F(t)
$$

Where,

$$
\left\{\begin{array}{c}
D^{k} F(t)=\left(m_{d}^{k}(t), \quad L_{d}^{k}(t), \quad R_{d}^{k}(t)\right) \\
m_{d}^{k}(t)=m_{d}^{k-1}(t+1)-m_{d}^{k-1}(t) \\
L_{d}^{k}(t)=\max \left\{L_{d}^{k-1}(t+1), \quad R_{d}^{k}(t)\right\} \\
R_{d}^{k}(t)=\max \left\{R_{d}^{k-1}(t+1), \quad L_{d}^{k}(t)\right\}
\end{array}\right.
$$

(6)

$D^{k} F(t)$, which is a fuzzy time series, is called the $k$ th order $(k=1,2.3, \Lambda)$ forward linguistic difference of $F(t)$ at $t$.

Then, we have:

$$
F(t+1)=F(t)+{ }_{w} D^{1} F(t-1)+{ }_{w} \Lambda+{ }_{w} D^{k} F(t-k)+{ }_{w} \Lambda
$$

If and only if for any $t \in T$, $m(t+1)=\sum_{i=0}^{\infty} m_{d}^{i}(t-i)$, where $m_{d}^{0}(t-0)=m(t)$, and

$$
L(t+1)=\max \left\{\sup _{k \leq t} L(k), \quad \sup _{k \leq t-1} R(k)\right\}
$$

$$
R(t+1)=\max \left\{\sup _{k \leq t} R(k), \quad \sup _{k \leq t-1} L(k)\right\}
$$

In a time variant model, the difference of the fuzzy time series at different times is determined by a window basis ( $k$ in formula (7)). The amount of a window basis determines how many of the previous data should be applied for the forecasting of a new value. The paper adopted a Genetic Algorithm (GA) to determine the value of $k$ in each time $t$.

\section{Genetic Algorithm procedure}

The GA procedure begins with determination of the population of chromosomes ${ }^{[12]}$. One evaluates these structures and allocate reproductive opportunities in such a way that those chromosomes, which represent a better solution to the target problem, are given more chance to "reproduce" than those chromosomes, which are poorer solutions.

\section{- Representation of the chromosomes.}

In order to encode each solution to a chromosome, one binary string is used for integer-valued variables. Population in our proposed algorithm incorporates 10 individuals, since this population size shows an appropriate performance.

\section{- Initial population generation.}

As the initial population, we generate population size chromosomes. Each individual chromosome in the initial population is a candidate solution to the problem, so for forecasting $F(t+1)$, it must have a value between 1 and $(t-1)$.

\section{- $\quad$ Fitness function.}

A commonly used transformation, which is applied in our proposed algorithm, is that of proportional fitness 
assignment. The individual fitness $F\left(x_{i}\right)$, of each individual is computed as the individual's raw performance, $f\left(x_{i}\right)$, relative to the whole population, i.e.,

$$
F\left(x_{i}\right)=f\left(x_{i}\right) / \sum_{i=1}^{N_{\text {ind }}} f\left(x_{i}\right)
$$

where, $N_{\text {ind }}$ is the population size and $x_{i}$ is the phenotypic value of the individual $i$.

- Crossover.

The single-point crossover is applied for this problem.

\section{- Selection.}

A roulette wheel selection procedure has been applied for the selection operator in our algorithm.

\section{- Mutation.}

Herr, the mutation probability is 0.001 .

\section{- Termination condition.}

In this algorithm method, we considered 200 generation to be termination condition.

\section{Defuzzification procedure}

This research adopted a back propagation Neural Network (NN) for the defuzzification. "Back-propagation" learning algorithm is an effective learning algorithm of multilayer perceptron and has directed attention to the information processing capability of artificial neural networks. This algorithm had been widely employed for various pattern classifications or inference problems expressed in terms of nonlinear functions ${ }^{[13]}$.

- The $k$-layer perceptron is a model consisting of one output layer and $(k-1)$ hidden layers.

- The I/O relationship of $\mathrm{NN}$ is expressed by the equation $y=N N(x)$, where $x$ and $y$ are input and output matrices.

- The term $k$-layers $\left[u_{0} \times u_{1} \times \Lambda \times u_{k}\right]$ expresses the model size, where $u_{i}$ is the number of neurons in the input layer, hidden layer(s), and output layer, and $k$ is the number of layers.

- $\quad$ Each input layer and hidden layer has an extra unit of constant 1 in addition to those specified by upside. This unit has no connection to the lower-layer neurons and has connections to the upper-layer neurons.

- All neuron models in the neighboring layers are connected, but there should be neither connections within layers nor jump connections between layers.
Here, a 5-layer back-propagation $\mathrm{NN}$ has been implemented. There are 5 neurons in each layer, except the output layer.

\section{Fuzzy variance and mean}

At the end of time period $t$, after its demand $\widetilde{D}_{k, t}$ has been realized, the participant observes the inventory level, places an order of size $\tilde{Y}_{k, t}$ to its supplier and receives this order at the beginning of time period $\left(t+\tilde{l}_{k}+1\right)$, where $\tilde{l}_{k}$ is the order lead time at stage $k$. Excess demand is backlogged ${ }^{[11]}$. We assume that the orders at any given stage become demands for the immediately upstream stage. Obviously we have $\widetilde{D}_{k+1, t}=\widetilde{Y}_{k, t}$.

Let $\widetilde{q}_{t}$ represent the ordered quantity in period $t$ to be delivered in period $(t+l)$, the timing of the event and the conservation of flow imply that:

$$
\widetilde{q}_{k, t}=\widetilde{Y}_{k, t}-\widetilde{Y}_{k, t-1}+\widetilde{D}_{t}
$$

It should be noted that all demands and lead times are considered to be uncertain and are represented by fuzzy triangular numbers.

We assume that each participant of the supply chain applies the same inventory and order policy as follows:

$$
\widetilde{D}_{k+1, t}=\widetilde{D}_{k, t}+\left(\widetilde{S}_{k, t}-\widetilde{S}_{k, t-1}\right)
$$

where, $\widetilde{S}_{k, t}$ is the fuzzy value of "the order-up-to level" at stage $k$ and period $t$.

$$
\widetilde{S}_{k, t}=\tilde{m}_{k, t}+z_{k} \sqrt{v_{k, t}}
$$

$$
\widetilde{m}_{k, t}=E\left(\sum_{i=1}^{l_{k}+1} \widetilde{D}_{k, t+i} \mid \widetilde{D}_{k, t}\right)
$$

$$
v_{k, t}=\operatorname{var}\left(\sum_{i=1}^{l_{k}+1} \widetilde{D}_{k, t+i} \mid \widetilde{D}_{k, t}\right)
$$

$$
z_{k}=\phi^{-1}\left(h_{k} /\left(p_{k}+h_{k}\right)\right)
$$

In this case, all the demands are considered to be fuzzy sets. The fuzzy number $\sum_{i=1}^{l_{k}+1} \widetilde{D}_{k, t+i} \mid \widetilde{D}_{k, t}$ can be extracted by using extension principle and forecasting of fuzzy demand from time $t$ to time $\left(t+\widetilde{l}_{k}+1\right)$, according to known demand at time $t^{[14]}$.

\section{Simulate the bullwhipeffect}

As described before, end customer's demand is generated by using an ARIMA process, and then these 
values are fuzzified into some symmetric triangular fuzzy numbers. These fuzzy values are considered as demands. The proposed approach is used for forecasting purposes. Since any lead time also has a fuzzy value, when computing the value of $\tilde{m}_{k, t}$ by using extension principle and cutting the $\tilde{l}_{t}$ in $\alpha$ level, the value of $\tilde{m}_{k, t}$ can become a type-II fuzzy set. To overcome this problem, a defuzzification on the values of $\sum_{i=1}^{l_{k}+1} \widetilde{D}_{k, t+i} \mid \widetilde{D}_{k, t}$ is implemented, by using the centroid method. Thus,

$$
\text { Bullwhip effect }=\operatorname{var}\left(q_{t}\right) / \operatorname{var}(D)
$$

\section{Discussion}

The characteristic ascending slightly in energy market shows China's energy supply being in shortage. Generally speaking, the energy consumers may keep the energy amount by increasing one and reducing the other in order to keep their utility steady under the situation of an adequate supply in energy market. To assess the performance of proposed uncertainty-dependent coal supply chain method and to gain further insights into the dynamical characteristics of supply chain systems with order placement lead time delays in an uncertain environment, especially the bullwhip phenomenon, we carry out simulations in this section.

Fig.1 Comparison of supplier's order and distributor's demand

We adopt a simulation example of the bullwhip phenomenon between coal supplier's order and coal distributor's demand in Xi'an city to demonstrate the feasibility and practicability of the proposed model. The occurrence of the bullwhip is shown in Figs. 1. Results showed that bullwhip still exists in such an environment. Nevertheless, it is clear that the variance of demand is amplified in supply chain when demand is transferred from downstream to upstream stages. The model can also forecast the trends in fuzzy data.

The previous approaches for overcoming the bullwhip effect did not consider fuzziness of environment. Based on above simulation, we concluded that the model can forecast the trends in fuzzy data. Adoption of the forecasting model can reduce the bullwhip effect and help in improving the customer service level with decrease in total cost of the coal supply chain. The model can suggest the reasonable ordering policies. The results also show that the propose model is superior to the previous analytical methods in terms of discovering the best available ordering policies.

\section{Conclusions}

In this paper, we have studied the bullwhip effect problem in uncertain coal supply chain systems. We proposed a model to simulated bullwhip effect in a fuzzy environment, when all demand and lead times were considered to be fuzzy numbers. Taking into consideration the multiple sources of uncertainty in supply chain systems, we focused on uncertainties with respect to demand and production process. Simulation study indicates that order placement do play an important role in reducing supply chain stability and inducing the bullwhip effect phenomenon. The larger the uncertain range of order placement is, the larger the inventory deviation amplitude is and the longer the bullwhip phenomenon exists.

It is important to note that our model is open-ended. There are many avenues for future research. Extending the proposed model to more detailed study, such as intensive study of the relationship between different uncertainty sources in coal supply chain operation process, is currently under investigation.

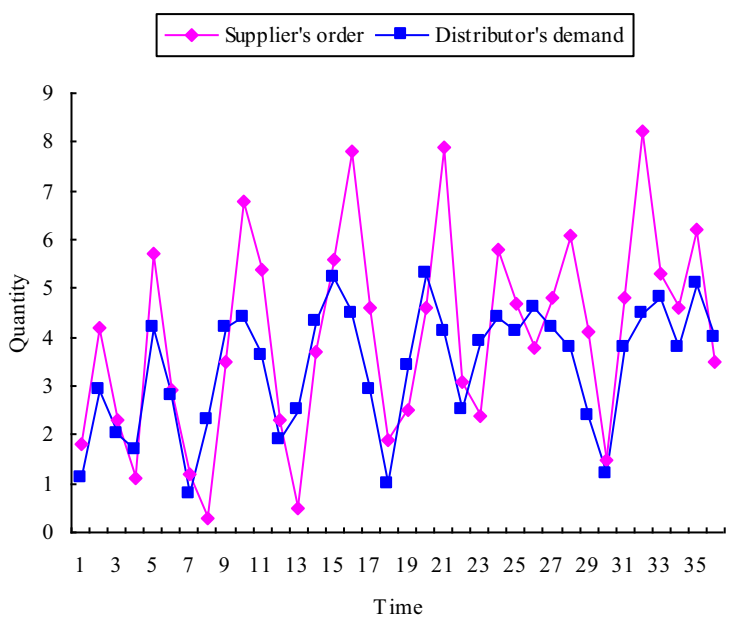

\section{Acknowledgment}

The research presented in this paper was supported by the Natural Science Foundation of China (NSFC) through Grant Nos. 71273206 and by the prosperity and development of philosophy and Social Sciences in Xi'an University of Science \& Technology under the grant numbers: 2016SY04.

\section{References}

[1] Ouyang, Y., Daganzo, C. F., Characterization of the bullwhip effect in linear, time-invariant supply chains: Some formulae and tests. Management Science. 52 (2006)1544-1556.

[2] Lee, H. L., Padmanabhan, V., Whang, S., The Bullwhip effect in supply chains. Sloan Management Review Spring. 38 (1997)93-102.

[3] Sterman, J. D., Modeling managerial behavior: misperceptions of feedback in a dynamic decision 
making experiment. Management Science. 35 (1989)321-339.

[4] Graves, S.C., A single-item inventory model for a non-stationary demand process. Manufacturing and Service Operations Management. 1 (1999)50-61.

[5] Zhang, X., The impact of forecasting methods on the bullwhip effect. International Journal of Production Economics. 88(2004)15-27.

[6] Chen, F., Drezner, Z., Ryan, J.K., Simchi-Levi, D., Quantifying the bullwhip effect in a simple supply chain. Management Science. 46(2000)436-443.

[7] Chen, Y.F., Disney, S.M., The order-up-to policy "sweet spot"- using proportional controllers to eliminate the bullwhip problem. In: EUROMA POMS Conference. 2(2003)551-560.

[8] Lee, H.L., So, K.C., Tang, C.S., The value of information sharing in a two-level supply chain. Management Science. 46(2000)626-643.

[9] M.S. Pishvaee, M. Rabbani, S.A. Torabi. A robust optimization approach to closed-loop supply chain network design under uncertainty, Appl. Math. Model. 35(2011)637-649.

[10] Hong, D. H., Some results on the addition of fuzzy intervals. Fuzzy Sets and Systems. 122(2001)349-352.

[11] Hong, D. H., A note on fuzzy time-series model. Fuzzy Sets and systems. 155(2005)309-316.

[12] M.H. Fazel Zarandi, M. Pourakbar, I.B. Turksen A, Fuzzy agent-based model for reduction of bullwhip effect in supply chain systems. Expert Systems with Applications. 34(2008)1680-1691.

[13] Takagi, H., Hayashi, I., NN-driven fuzzy reasoning. International Journal of Approximate Reasoning. 5(1991)191-211.

[14] Carlsson, C., Fuller, R., On possibilistic mean value and variance of fuzzy numbers. Fuzzy Sets and Systems. 122(2001b)315-326. 